\title{
Les conflits interculturels dans les entreprises mixtes sino-françaises et propositions de solution
}

\section{Zhang Jinhai et Yao Xi}

\section{(2) OpenEdition}

12 Journals

Édition électronique

URL : http://journals.openedition.org/communicationorganisation/3433

DOI : 10.4000/communicationorganisation.3433

ISSN : 1775-3546

Éditeur

Presses universitaires de Bordeaux

\section{Édition imprimée}

Date de publication : 1 juin 2006

Pagination : 270-281

ISSN : 1168-5549

\section{Référence électronique}

Zhang Jinhai et Yao Xi, « Les conflits interculturels dans les entreprises mixtes sino-françaises et propositions de solution », Communication et organisation [En ligne], 29 | 2006, mis en ligne le 21 juin 2012, consulté le 18 décembre 2020. URL : http://journals.openedition.org/

communicationorganisation/3433; DOI : https://doi.org/10.4000/communicationorganisation.3433

Ce document a été généré automatiquement le 18 décembre 2020.

(C) Presses universitaires de Bordeaux 


\title{
Les conflits interculturels dans les entreprises mixtes sino-françaises et propositions de solution
}

\author{
Zhang Jinhai et Yao Xi
}

1 La France est un des pays industriellement développés : elle occupe la quatrième place sur le plan économique mondial, 25 entreprises françaises se classent parmi les 500 premières puissances du monde. En 2000, les investissements vers l'extérieur de la France ont dépassé pour la première fois ceux des Etats-Unis, elle est devenue ainsi le deuxième investisseur derrière la Grande Bretagne. Les entreprises françaises sont également parmi les premières entreprises étrangères implantées en Chine. En 1973, la France et la Chine ont signé une convention de coopération concernant l'Usine de la fibre chimique de Liaoyang, qui était le plus grand projet de coopération industrielle de la Chine à l'époque. En 1980, le groupe français Rémy-Martin a créé une entreprise à capitaux mixtes à Tianjin, Sino-French Joint-Venture Dynasty Winery Ltd, qui est la deuxième entreprise mixte en Chine. Depuis plus de vingt ans, la coopération entre les entreprises françaises et chinoises a connu de grands succès, les échanges économiques et commerciaux entre les deux pays se sont intensifiés rapidement. Depuis 1996, les investissements annuels français au continent chinois sont de 1500 millions d'euros.

De janvier à novembre 2003, le volume du commerce entre les deux pays a augmenté de $60 \%$ par rapport à l'année précédente, passant de 8,33 milliards de dollars en 2002 à 11,9 milliards de dollars en 2003. La Chine a introduit en 1972 des projets technologiques Français, dont le valeur est de 10,9 milliards de dollars, et qui ont occupé la deuxième place au sein de l'Union Européenne. Récemment, les entreprises françaises ont élargi leurs investissements dans les régions centrales et ouest de la Chine, la France est devenue désormais le troisième pays investisseur européen de la Chine. Ce pays occupe indubitablement une place très importante sur le plan de l'investissement.

3 Mixer les capitaux est la forme de coopération économique internationale la plus pratiquée dans le monde d'aujourd'hui. Une entreprise à capitaux mixtes comporte un 
ensemble de capitaux, de technologies, de ressources humaines et de travail. Son objectif est un échange et une confrontation culturels entre les partenaires, voire une fusion de leur culture d'appartenance. Les chefs d'entreprise se trouvent dans un environnement entièrement différent de leur environnement d'origine, et se confrontent à des notions de valeurs et des règles de conduite spécifiques dans cet environnement. Ils ont donc de nombreux problèmes à résoudre, qui ne se limitent pas à des problèmes comme l'organisation de l'entreprise, les ressources humaines et l'orientation de l'investissement, mais abordent aussi des contradictions et des conflits causés par les différences culturelles dans ce contexte interculturel.

4 Au sein d'une entreprise mixte, les différences culturelles entre les deux parties coopérantes se manifestent dans des aspects comme : les cultures héritées, les notions de valeur, les environnements géographiques, les croyances religieuses et les systèmes institutionnels. Dans un certain sens, ce croisement culturel provoquera des conflits culturels inévitables, ceux-ci deviendront un des obstacles de l'expansion de l'entreprise. Selon certaines études, il est prévu que $15 \%$ des entreprises mixtes en Chine aboutissent à l'échec prématurément, cet échec étant principalement dû à l'instabilité de l'entreprise, qui est entrainée par les conflits culturels. Parmi les nombreuses entreprises françaises, certaines comme Alsthom, Airbus et Carrefour ont connu des succès, mais les exemples d'échec ne manquent pas.

5 Ainsi, nous avons centré notre recherche sur la problématique interculturelle existant dans les sociétés mixtes sino-françaises. Dans les parties qui suivent, nous analyserons d'abord des cas particuliers de conflit interculturel dans ces entreprises afin de mettre en lumière ce qui se cache derrière ses conflits. Par la suite, nous nous efforcerons de faire des recherches en vue de trouver une solution. Il faut noter que notre travail n'est pas une simple étude sur les conflits interculturels dans les entreprises mixtes sinofrançaises, qu'il fournit également des pistes de référence pour résoudre les problèmes interculturels et présente un modèle de recherche.

\section{La situation actuelle et ses caractéristiques}

6 Les recherches antérieures n'ont pas donné de statistiques précises des échecs ayant pour origine des conflits interculturels. L'analyse de certains échecs des entreprises telle que Peugeot à Guangzhou, nous permet à dégager la situation actuelle et révéler les caractéristiques de ces conflits.

7 Le 26 septembre 1985, Peugeot a créé sa première société mixte en Chine GuangZhou Peugeot Automobile (GPAC). C'est une société à capitaux mixtes, contrôlée conjointement par la société Guangzhou Automobile ( 46 \% de contrôle), Automobile Peugeot ( 22 \% de contrôle, dont la principale est l'investissement technologique), la société de crédit et d'investissement international de la Chine ( $20 \%$ de contrôle), la société de finance international ( $8 \%$ de contrôle) et Banque nationale de Paris (BNP : $4 \%$ de contrôle). L'équipe de la société Guangzhou Automobile compte 2000 personnes, administrées conjointement par cette société et Peugeot. Jusqu'en août 1997, Guangzhou Automobile se trouvait devant un déficit total de 1,05 milliards de RMB, sa maximum production annuelle réelle était de 21000 véhicules, loin de ce que la politique industrielle de l'Etat a exigé, soit de 150000 véhicules. En même temps, la partie chinoise et la partie française avaient de grandes divergences de vue sur des problèmes importants, la coopération ne pouvait pas se poursuivre ainsi. Une convention a été signée par les 
deux pays en septembre 1997, qui a mis la fin à la coopération entre Peugeot et la société Guangzhou Automobile.

Juste après ce divorce, San Jiang Renault est engagé aussi dans une séparation. Cette entreprise mixte automobile a été créée en novembre 1997 dans la ville de Xiangfan de la province du Hubei, par le Groupe chinois San Jiang et Renault, contrôlée à $55 \%$ par le groupe chinois et $45 \%$ pour la partie française. Ils coopéraient pour assembler et produire des Trafic (bus léger), pour un délai de trente ans. Néanmoins, de 1994 à 2001, on n'a vendu que 4100 Trafic. Au cours de cette coopération, il y avait des désaccords sérieux entre les Chinois et les Français sur les cultures et les stratégies. Donc, le groupe San Jiang s'est séparé à partir de 2000. Par la suite, la société San Jiang Renault a changé quatre fois de propriétaire.

Un autre cas remarquable est celui de la coopération entre Dong Feng Automobile et Citroën. Cette coopération remonte aux années 90 , où le groupe PSA et le groupe automobile Dong Feng ont signé un accord de partenariat. Mais durant les dizaines d'années de coopération, on a toujours constaté des atmosphères peu harmonieuses. En tant que la première société mixte en Chine du groupe PSA, en 2004 Dong Feng Peugeot Citroën Automobile (DPCA) connaît une baisse de $13,8 \%$ de part du marché, et réalise un chiffre d'affaire de 8491 milliards de RMB, en baisse de 21,9\% par rapport à l'année précédente. La société a déclaré un déficit de 600 millions de RMB. A vrai dire, le déficit de DPCA est dû à plusieurs raisons, y compris les conflits interculturels à l'origine des divergences culturelles. Il existe toujours des fissures entre la culture chinoise, une culture très ancienne, et la culture française, une culture extrêmement romantique, disent certains journalistes étrangers.

10 Selon notre recherche sur certaines entreprises sino-françaises, les conflits interculturels se manifestent principalement dans les aspects suivants.

\section{La politique des ressources humaines}

11 En ce qui concerne l'administration du personnel, les Chinois mettent l'accent sur la qualité politique, les antécédents et les relations humaines des employés, tandis que les Français donnent une place importante à la rationalité de l'administration personnelle, qui met en rapport le remaniement personnel, les responsabilités du poste ainsi que le résultat du travail. Ces différences de normes sur les ressources humaines engendrent inévitablement des malentendus entre les deux parties, et constituent des aspects défavorables au développement de l'entreprise. Quant au système de salaire, selon les Chinois, le salaire doit être payé selon des normes rigides, tels que: ancienneté, diplôme et antécédents, et se réajuster selon la rentabilité économique de l'entreprise. Par contre, d'après les Occidentaux, il faut mettre le salaire en liaison avec la position $\mathrm{du}$ poste et le résultat du travail, et le réajuster selon l'indice du coût de la vie. L'opposition entre ces deux concepts différents, non seulement sème des troubles à l'élaboration et l'exécution $\mathrm{du}$ système de salaire, mais aussi soulève du mécontentement de certains employés et exerce ainsi de mauvaises influences sur la relation et la coopération sino-françaises dans l'entreprise. 


\section{Le mode de l'administration}

Les Français préfèrent une administration de l'entreprise régulière et réglementée. Ils ont l'habitude d'administrer et gérer une entreprise dans un contexte où la légalité est précise. Dans une entreprise occidentale, depuis l'élaboration de la stratégie jusqu'au management, tous les processus sont strictement règlementés. Alors que les administrateurs chinois attachent plus d'importance au sentiment humain. Ceux-ci s'habituent à suivre la volonté des supérieurs hiérarchiques et aux directives de l'administration supérieure. Ils déclinent souvent les responsabilités et manquent d'initiative. Ces habitudes des Chinois nuisent à l'efficacité de leur travail, entrainent des désaccords avec les Français. En résumé, les volontés de coopération de ces deux parties sont affaiblies à cause de ces différences concernant le mode d'administration.

\section{Le concept de gestion}

13 Par rapport à l'entreprise chinoise, l'entreprise française insiste plus sur la rentabilité à long terme, tandis que l'entreprise chinoise prête plus d'attention aux actions à court terme. Quant à leur style de gestion, les directeurs français décident personnellement et prennent la responsabilité de leur décision, alors que les Chinois ont l'habitude de décider en groupe et de partager aussi la responsabilité. Une philosophie traditionnelle chinoise est que l'on souhaite la stabilité et craint la mutation, sous l'influence de laquelle, les gestionnaires chinois manquent d'une prise de conscience du risque. Ils sont conservateurs dans leurs décisions, qui sont souvent trop tardives par rapport aux opportunités sur le marché. Par contraste, les administrateurs français osent les innovations et prennent des risques, par exemple, ils adoptent de nouvelles technologies ou exploitent de nouveaux marchés.

Si les entreprises sino-françaises ne peuvent pas prévenir et résoudre les problèmes interculturels, elles vont les payer cher, parce que ces derniers leur coûteront non seulement de l'argent, mais aussi du temps et des efforts.

\section{Les origines des conflits interculturels}

15 L'entreprise sino-française connaît une situation complexe à l'extérieur, et des cultures différentes coexistent à l'intérieur. Depuis sa création à la gestion, il existe toujours des conflits. Certains ont pour origine de la différence entre la culture chinoise et la culture de l'entreprise mixte, les autres sont dues à des désaccords entre les Français et les Chinois appartenant à deux groupes culturels.

16 Ce qui se cache derrière toutes sortes de phénomènes conflictuels au sein de l'entreprise sino-française, ce sont les différences culturelles entre les deux pays. Bien que la France ait plus d'éléments similaires à la Chine par rapport à d'autres pays occidentaux, il existe réellement des divergences non négligeables entre ces deux pays. Elles se reflètent dans leurs notions de valeur, de culture institutionnelle, de mode de planification et d'élaboration stratégique, d'administration du personnel et de mode de pensée. 


\section{Notion de valeur}

17 Représentée par la culture chinoise, la culture orientale se développe sur la base du confucianisme. Elle est une culture agricole, qui est en même temps une culture patriarcale fondée sur la base de la relation patriarcale et consanguine. C'est pourquoi la culture orientale a de l'estime pour la collectivité, le sens moral et la pratique. Elle se caractérisée particulièrement par son indépendance et son introversion. La personnalité occidentale est fondée sur la culture religieuse occidentale et la civilisation commerciale. Elle est caractérisée par son autonomie, son individualisme et son ouverture, ainsi que sur l'égalité et la démocratie dans la société où elle se trouve. L'égalité, la liberté et la fraternité dans la culture française font partie de l'esprit humaniste, qui donne une place importante à la subjectivité de l'être humain. Dans l'ensemble, les Français ont un fort esprit individuel.

\section{Culture institutionnelle}

Dans l'entreprise chinoise, la volonté humaine compte beaucoup et on observe une flexibilité dans l'exécution des règlements. La culture orientale est la culture ayant la plus grande vitalité, une des principales raisons de sa flexibilité et son adaptabilité. Cependant, il est certain que la flexibilité excessive engendre la sous-estimation de la réglementation, dans le cas-là, on prend souvent des mesures en fonction de différents paramètres. Donc, dans une entreprise chinoise, le respect d'un règlement est parfois mis à mal, pour un cas exceptionnel, sous les directives d'un supérieur hiérarchique. Quant à l'entreprise française, elle se trouve dans une économie du marché développé, elle a des systèmes et des règlements rigides pour son administration. Son mécanisme organisationnel est perfectionné, chaque échelon est chargé de son administration particulière et précise ; la division du travail et la coopération sont soulignées.

\section{Mode de planification et d'élaboration stratégique}

Dans la culture d'entreprise chinoise, la planification et l'élaboration stratégique sont un processus complexe et long. En général, ce processus est soumis à de nombreuses formalités, allant de la discussion à plusieurs reprises de la promulgation de toutes directives. Ce mode d'élaboration est caractérisé particulièrement par la décision collective, dont l'avantage est de mettre à profit la sagesse collective et d'obtenir une grande exactitude. Son point faible est sa réaction lente au changement du marché. Par contre, on observe un autre mode d'élaboration stratégique dans l'entreprise française : le pourvoir est concentré, la réaction au changement est rapide, mais avec moins d'exactitude et un manque de compétence concurrentielle à long terme.

\section{Ressources humaines}

On constate un esprit humaniste dans les cultures d'entreprise des deux pays, les entreprises chinoises et françaises respectent les employés, leurs créent un environnement de travail agréable dans le but de mobiliser leur esprit d'initiative et de créativité. Néanmoins, on perçoit des différences évidentes en ce qui concerne les 
rapports entre l'individu et la collectivité. L'esprit d'équipe dans l'entreprise française se base sur l'individualisme, l'individu occupe une place prioritaire. Il existe une relation d'égalité et d'entraide mutuelle entre l'individu et la collectivité. Par contre, la collectivité est toujours supérieure à l'individu dans l'entreprise chinoise, l'individu est subordonné à la collectivité et ne peut être reconnu et développé que dans la collectivité. Cela reflète donc un esprit de sacrifice.

\section{Mode de pensée}

21 La pensée chinoise est typiquement une pensée courbe, selon laquelle on se base d'abord sur des principes, pour prendre ensuite en considération les aspects concrets. Lorsque cette pensée s'applique aux affaires commerciales, elle se reflète dans le processus de résolution: les problèmes abstraits d'abords, les problèmes concrets ensuite, c'est-à-dire, discuter d'abord sur les principes en essayant de se mettre d'accord, résoudre ensuite les problèmes micro en fonction de ces principes. La pensée française est plus rationnelle, claire et directe. Très souvent, afin de résoudre un problème, les Français s'efforcent de trouver ou d'établir les rapports causals et logiques, sans lesquels ils n'arrivent pas à prendre la décision. Il leur semble que ce processus de recherche est plus important que le résultat. Prenons comme un exemple la discussion sur le budget et le développement, celle-ci est souvent centrée sur la déduction des liens causals entre certains indices. Cette déduction logique à la façon française ne peut pas s'exprimer par la formule mathématique "rentable ou non rentable ", et est très différente de la philosophie propre aux Chinois --- «la doctrine de juste milieu » (Zhengzhou). Pour les Français, une fois le lien logique établi, il change rarement. Ce mode de pensée ne pose pas de problèmes importants dans les organismes locaux, mais quand les Français continuent à agir de telle façon à l'étranger, ils rencontrent souvent des difficultés dans la confrontation à d'autres cultures ou logiques. L'échec de certaines entreprises françaises en Chine peut le prouver.

\section{Résolution}

Les différences précédemment exposées entre la culture chinoise et la culture française conduisent à des conflits interculturels entre les deux partenaires, lesquels se manifestent majoritairement dans la politique administrative, dans la collaboration organisationnelle, dans le mode gestionnaire. Ces conflits deviennent un des obstacles cruciaux dans le développement de l'entreprise, car ils détruisent l'efficacité du travail et la stabilité de l'entreprise. Donc, il est nécessaire de les identifier et d'y mettre fin. Ce travail permettrait le bon fonctionnement de l'entreprise mixte.

\section{Identifier les différences culturelles, reconnaître la culture étrangère par l'intermédiaire de la communication mutuelle}

Comme nous l'avons dit précédemment, les différences idéologiques dans l'administration sont à l'origine des divergences entre certaines valeurs des deux pays. Donc, pour faire disparaitre les mauvais effets de ces divergences, il importe que les 
administrateurs et les employés prennent conscience des divergences culturelles, qu'un nouveau mode d'administration soit mis en œuvre sur la base du respect mutuel. Voilà la condition préalable pour résoudre les problèmes interculturels. Une base sera de respecter la culture et le mode de pensée de l'autre, de reconnaître l'existence réelle des différences, d'éviter à tomber dans l'ethnocentrisme, et d'attacher de l'importance à l'apprentissage de la langue, de la culture, de l'économie de l'autre pays.

Initier le personnel de l'entreprise au respect et à la compréhension des différences culturelles, permettra de faire naître une reconnaissance culturelle chez tous les membres de l'entreprise mixte. C'est à partir de cette reconnaissance que se forme le mode d'administration spécifique à l'entreprise mixte, comprenant des modes de pensée, de travail et de communication particuliers. Il faut que les employés chinois et français exercent des échanges amicaux mutuels, car la reconnaissance se développe par le biais de la communication interculturelle.

\section{Etablir une notion de valeur commune et créer une culture institutionnelle partagée}

L'établissement d'une notion de valeur commune au sein de l'entreprise mixte rassemblera les forces pour la réalisation de l'objectif commun et mettra de coté les désaccords interculturels. Composante essentielle de la culture, cette notion de valeur s'établit au fur et à mesure comme une conviction permanente. Elle permet de repérer les critères pertinents pour améliorer les comportements et les relations humaines... Eviter l'ethnocentrisme, respecter la culture de l'autre, communiquer d'égal à égal, tel est la base pour repérer le point de combinaison des deux cultures, promouvoir leurs point forts et établir une notion de valeur commune. En même temps, une culture d'entreprise devrait être construite en fonction des exigences propres à l'environnement et à l'attente stratégique. Ainsi, une culture administrative se forme par le bais de l'échange culturel, suivi par la création d'une culture institutionnelle centrée sur la notion de valeur commune.

\section{Donner des formations interculturelles}

A l'heure actuelle, la majorité des entreprises soulignent l'importance des formations techniques, sans penser à la formation interculturelle auprès du personnel, notamment auprès des gestionnaires. Pourtant, celle-ci est le moyen le plus efficace et le plus élémentaire de résoudre les problèmes interculturels. Généralement, une formation interculturelle se compose de :

- Connaissance de la culture de son partenaire : elle est sous forme de séminaire, cours, formation linguistique, livre, site Internet, discussion, jeu de rôle, etc.

- Sensibilité culturelle : développer leur compétence à analyser les caractéristiques de la culture du partenaire, afin de comprendre comment sa culture d'appartenance détermine son comportement et de saisir les composantes élémentaires de la culture étrangère.

- Compétence d'adaptation culturelle : envoyer l'employé à l'étranger en mission pour qu'il vive la vie des autres, cela permet également à l'employé de développer sa capacité d'adaptation à une culture étrangère. 
- Langue étrangère : la langue est une composante importante de la culture, la communication linguistique est le moyen le plus efficace pour améliorer la compétence d'adaptation culturelle. Cette formation vise non seulement à développer leur compétence linguistique, mais aussi à connaître certains modes spécifiques de communication dans un pays étranger.

- Communication interculturelle : mettre en place des organisations officielles ou non officielles de la communication interculturelle.

\section{Localisation du mode de planification et d'élaboration stratégique}

Il est approprié d'exercer une administration interculturelle en s'appuyant sur le principe "globaliser la pensée, localiser les actions». Au lieu d'imposer le mode d'administration de la société mère, il est plus pertinent de faire référence à celui de l'entreprise chinoise. La combinaison de l'exactitude chinoise avec la rapidité française fournira une base d'élaboration stratégique de l'entreprise mixte sino-française. En général, en ce qui concerne les politiques stratégiques importantes, il est convenable de recueillir l'avis de tous et de mobiliser pleinement l'initiative des employés pour que ces politiques soient exactes et pertinentes; quant aux stratégies peu importantes, les responsables de chaque hiérarchie pourront prendre la décision de façon rapide et flexible.

\section{Administration interculturelle des ressources humaines}

Puisque les divergences culturelles se manifestent dans la pensée, la valeur et le comportement des êtres humains, l'organisation culturelle est en effet une organisation des notions de valeur. Pour résoudre les problèmes interculturels, on a besoin d'administrateurs de haute compétence interculturelle, souples, réactifs, possédant une capacité d'adaptation et le sens de l'égalité et du respect, qui puissent supporter les avis divergents et sachent vivre au contact de gens issus d'autres groupes culturels. L'administration interculturelle au sein de l'entreprise mixte devrait mettre l'accent sur l'administration des ressources humaines, les administrateurs doivent comprendre la culture de la société mère. En même temps, il faut renforcer l'administration culturelle du personnel, de sorte que la nouvelle culture d'entreprise exerce ses effets significatifs, que l'entreprise mixte l'emporte dans la concurrence avec les entreprises étrangères.

\section{Conclusion}

Actuellement, de plus en plus d'entreprises françaises sont attirées par de bonnes perspectives et la grande potentialité du marché chinois, elles accélèrent le pas pour les investissements en Chine. Les entreprises sino-françaises sont en pleine expansion. Néanmoins, les conflits interculturels constituent un grand obstacle dans leur développement, qui peut même aboutir à l'échec. Dans ce contexte, les recherches portant sur la problématique interculturelle existant dans l'entreprise sino-française 
ont une pertinence actuelle. Cependant, l'insuffisance de recherches référentielles ne nous donne pas la possibilité de développer en détail notre travail. Nous espérons que nos collègues chinois et français pourront approfondir la recherche portant sur les conflits interculturels.

\section{BIBLIOGRAPHIE}

Darlington, J., Culture : théorie et administration interculturelle, Maison d'édition de finance de Dongbei, 1998.

Barsoux, J.-L., Schneider C., Administration interculturelle, Maison d'édition de gestion économique, 2002.

Meloan W., Taylor, Marketing interculturel, Maison d'édition d'industrie mécanique, 1998.

Weiying Z., Culture : normes comportementales et conviction commune d'un groupe, Maison d'édition de San Lian, 2000.

Zhang, Xinsheng et Wang, Yuan, Management international, Maison d'édition de l'université du peuple, 2002.

Yuan, Yijun, Management de l'entreprise internationale, Maison d'édition de l'université technologique et scientifique de Dalian, 199.

Zhao, Shuming ; Yang, Zhong, Entreprise internationale : administration interculturelle, Maison d'édition de l'université de Nanjing, 1994.

Li, Ye et He, Qianyin, « Guangzhou Peugeot, conflit et fusion des cultures différentes ", in Culture d'entreprise chinoise et étrangère, $\mathrm{n}^{\circ} 1$, pp. 16-18, 2000.

\section{RÉSUMÉS}

Après l'ouverture vers l'extérieur, grâce à son environnement d'investissement bien organisé et à la rapidité de sa croissance économique, la Chine se classe parmi les pays ayant le plus de capacités à attirer l'investissement étranger. En tant que troisième investisseur européen, la France occupe indubitablement une place très importante dans l'investissement étranger en Chine. Parmi les nombreuses entreprises sino-françaises, certaines ont connu le succès en Chine, mais les échecs ne manquent pas. Les conflits interculturels sont un des obstacles élémentaires dans le développement de l'entreprise mixte. Dans ce contexte, les recherches portant sur la problématique de l'interculturel existant dans l'entreprise sino-française ont une pertinence certaine. Actuellement, de plus en plus d'entreprises françaises sont attirées par des perspectives intéressantes et la grande potentialité du marché chinois, elles accélèrent le pas pour l'investissement en chine. 
INDEX

Mots-clés : entreprise mixtes sino-françaises, conflit interculturel

\section{AUTEURS}

\section{ZHANG JINHAI}

Institut du Journalisme et de la Communication - Université de Wuhan

YAO XI

Institut du Journalisme et de la Communication - Université de Wuhan 